\title{
ARTICLE miRNAs in mtDNA-less cell mitochondria
}

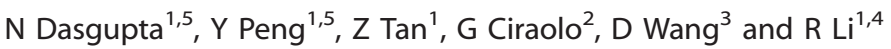

The novel regulation mechanism in mtDNA-less cells was investigated. Very low mtDNA copy in mtDNA-less $206 \rho^{\circ}$ cells was identified. But no 13 mitochondria-specific proteins were translated in $206 \rho^{\circ}$ cells. Their mitochondrial respiration complexes $\mathrm{V}$, III and II were 86.5, 29.4 and 49.6\% of 143B cells, respectively. Complexes I and IV completely lack in $206 \rho^{\circ}$ cells.

Non-mitochondrial respiration to generate ATP in $206 \rho^{\circ}$ cells was discovered. The expression levels of some mitochondrial RNAs including 12S rRNA, COX1, COX2, COX3, ND4 and ND5 were low. However, ND1, ND3 and Cyto b were not expressed in $206 \rho^{\circ}$ cells. Unequal transcription of mitochondrial RNAs indicated the post-transcriptional cleavage and processing mechanisms in the regulation of mitochondrial gene expression in $206 \rho^{\circ}$ cells. MicroRNAs (miRNAs) may modulate these mitochondrial RNA expression in these cells. RNA-induced silencing complex indeed within $206 \rho^{\circ}$ cell mitochondria indicated miRNAs in $206 \rho^{\circ}$ cell mitochondria. miRNA profile in mtDNA-less $206 \rho^{\circ}$ cells was studied by next-generation sequencing of small RNAs. Several mitochondria-enriched miRNAs such as miR-181c-5p and miR-146a-5p were identified in $206 \rho^{\circ}$ cell mitochondria. miR-181c-5p and miR-146a-5p had 23 and 19 potential targets on mitochondrial RNAs respectively, and these two miRNAs had multiple targets on mitochondria-associated messenger RNAs encoded by nuclear genes. These data provided the first direct evidence that miRNAs were imported into mitochondria and regulated mitochondrial RNA expressions.

Cell Death Discovery (2015) 1, 15004; doi:10.1038/cddiscovery.2015.4; published online 27 July 2015

\section{INTRODUCTION}

MicroRNAs (miRNAs) regulate gene expression via partial base pairing within RNA-induced silencing complex (RISC) to their target messenger RNA (mRNAs) for degradation or suppression of mRNA translation. The precursors of miRNAs are small non-coding RNA molecules. miRNAs are transcribed as $\sim 70$ nucleotide precursors and subsequently processed by the RNase-III type enzyme Dicer to give a 18- to 25-nucleotide mature product. The mature miRNA is part of an active RISC containing Dicer and many associated proteins. Members of the Argonaute protein family are central to RISC function. Argonaute 2 (Ago2) is the key component of RISC. ${ }^{1-3}$ In general, miRNA are highly conserved in different species, and are important regulators of gene expression in human cells. An explosion of interest has led to $\sim 2000$ mammalian miRNAs being described. miRNAs have been shown to have critical roles in many cellular and disease processes. ${ }^{4-7}$ Several miRNAs have been shown to be imported into mitochondria and involved in the regulation of mitochondrial metabolism. ${ }^{8,9}$ Characterizing the expression profile of miRNAs in mitochondria is a first step toward understanding their role in mitochondrial gene regulation.

Mitochondria, supplying $90 \%$ of energy for cells, are also involved in many other essential cellular processes, such as creating reactive oxygen species, regulating apoptosis and buffering calcium. ${ }^{10}$ More than 1500 proteins are found in the mitochondria. ${ }^{11}$ The majorities of mitochondrial proteins are products of nuclear genes and are synthesized in the cytosol, then imported into the mitochondria. The mitochondrial genome in mitochondria codes for only 13 peptides/proteins, 22 tRNAs and
2 ribosomal RNAs. Thirteen peptides encoded by mitochondrial genome (mtDNA) are components of respiratory chain complexes located on the inner membrane of mitochondria.

It is believed that 143B-derived mtDNA-less cells by ethidium bromide (EtBr) treatment, $206 \rho^{\circ}$ cells, have no mitochondrial genome, which could not transcript to mitochondrial RNAs and are unable to translate 13 mitochondria-specific proteins. ${ }^{12}$ It has not reported that the effect of miRNAs in mtDNA-less $206 \rho^{\circ}$ cells. This article describes a study of miRNA profile in mtDNA-less $206 \rho^{\circ}$ cell mitochondria to elucidate the regulation mechanism of miRNAs on mitochondrial RNAs in mtDNA-less cells.

\section{RESULTS}

It was demonstrated that miRNAs regulated gene expression by targeting mRNAs through partial base-pairing complementary sites in cytosol. It was reported that some miRNAs were present in the mitochondria of liver cells, human myoblasts and HeLa cells. ${ }^{8,9,13}$ But the significance and functional consequences have not been characterized. The study is to determine whether miRNAs, such as proteins, could translocate into the mitochondria and regulate mitochondrial gene expression in $206 \rho^{\circ}$ cells.

mtDNA-less, not mtDNA lack, in $206 \rho^{\circ}$ cells

Usually, it is accepted that $206 \rho^{\circ}$ cells is an mtDNA-lacking cell line. To detect whether $206 \rho^{\circ}$ cells contain mtDNA, DNA samples from $143 \mathrm{~B}$ and $206 \rho^{\circ}$ cells were amplified in 24 overlapping fragments by use of 24 pairs of oligonucleotide primers. ${ }^{14}$ We found that some mitochondrial genomic regions in $206 \rho^{\circ}$

${ }^{1}$ Division of Human Genetics, Cincinnati Children's Hospital Medical Center, 3333 Burnet Avenue, Cincinnati, OH 45229, USA; ${ }^{2}$ Division of Pathology, Cincinnati Children's Hospital Medical Center, 3333 Burnet Avenue, Cincinnati, OH 45229, USA; ${ }^{3}$ Maxillofacial Pathology and Radiology Department, Ohio State University College of Dentistry, 304 W. 12 th, Columbus, $\mathrm{OH} 43210, \mathrm{USA}$ and ${ }^{4}$ Department of Pediatrics, University of Cincinnati College of Medicine, Cincinnati, $\mathrm{OH} 45229$ USA

Correspondence: R Li (Ronghua.Li@cchmc.org) or D Wang (wang.7277@osu.edu)

${ }^{5}$ These authors contributed equally to this work.

Received 4 June 2015; accepted 5 June 2015; Edited by G Melino 
including primers 2, 8, 9 11, 12, 13 and 19 (Supplementary Figure S1) had the same PCR amplicons as $143 B$ cells. After sequencing these PCR amplicons and BLAST homology searches on National Center for Biotechnology Information (NCBI) website, $^{15}$ we confirmed that these sequences of PCR products from $206 \rho^{\circ}$ were same as $143 \mathrm{~B}$ mtDNA genome including mitochondrial ribosome (12S rRNA and 16S rRNA) genes, and genes of ND2, COX1, COX2, A6 and ND5.

To determine the relative DNA copy numbers of $206 \rho^{\circ}$ cells to $143 B$ cells, qPCR was carried out to determine the ratio of mtDNA $\mathrm{HV} 1$ region to nucleus-encoded $\mathrm{H} 3$ gene. The results demonstrated that mtDNA copy numbers in $206 \rho^{\circ}$ was $1 / 164$ of mtDNA in $143 B$ cells.

Partial expression of mitochondrial RNAs

To examine whether $206 \rho^{\circ}$ mtDNA transcript mitochondrial mRNA, RT-PCR was performed using cDNA from $206 \rho^{\circ} \mathrm{mt}-\mathrm{R}$ RNA sample. The results demonstrated that some mitochondrial RNAs were expressed in $206 \rho^{\circ}$ cells (Supplementary Figure S1) including 125 and $16 \mathrm{~S}$ rRNA, ND2, COX1, COX2, A6, ND4, ND4L, ND5 and ND6 RNAs. But the expression levels of these mitochondrial RNAs were lower than in 143B cells. And mitochondrial mRNAs of ND1, ND3 and Cytb were not expressed in $206 \rho^{\circ}$ cells (Figure 1). RT-qPCR results confirmed that the expression levels of ND1, ND3 and Cyto b mRNAs were extremely low.

Lack of mitochondria-specific protein synthesis

The mitochondrial genome encodes for 37 genes: 2 rRNAs (12S and $16 \mathrm{~S}$ rRNA) and 22 tRNAs required for mitochondrial protein synthesis and 13 mitochondria-synthesized peptides essential to the respiratory chain complexes. The results of $\left[{ }^{35} \mathrm{~S}\right]-$ pulse-labeling mitochondrial proteins in vitro demonstrated that 206 mtDNA-less $\rho^{\circ}$ cells did not translate 13 mitochondria-specific peptides/proteins (Supplementary Figure S2), even though some mitochondrial genomic regions were detected and some mitochondrial mRNAs were partially expressed in $206 \rho^{\circ}$ cells.

Decreased expression of mitochondrial complexes

Oxidative phosphorylation is an important cellular process that uses oxygen and simple sugars to produce ATP. Five protein complexes (complexes I-V), made up of several proteins encoded by nuclear gene or/and mitochondrial genomic genes, are involved in this process. The protein ratios of mitochondrial genomic gene encoded to unclear gene encoded in complexes I-V are $7 / 37,0 / 4,4 / 25,3 / 16$ and $2 / 17$, respectively. Our western blot results showed the significantly decreased amounts of complexes II (29.4\%) and III (49.6\%) compared with 143B cells, and complexes I and IV lacked completely in $206 \rho^{\circ}$ cells (Supplementary Figure S3). The antibody to probe complex IV is anti-COX1. Complex IV completely lost in western blotting confirmed no mitochondrial COX1 were synthesized in $206 \rho^{\circ}$ cells by ${ }^{35} \mathrm{~S}$ pulse labeling of mitochondrial protein synthesis even though partially expressed COX1 mRNA (Figure 1). But complex V kept high expression in $206 \rho^{\circ}$ cells. The expression of complex $\mathrm{V}$ in $206 \rho^{\circ}$ cells was $86.5 \%$ when compared with that of $143 \mathrm{~B}$ cells.

\section{Marked decreased mitochondrial respiration}

No mitochondria-specific 13 peptides/proteins were synthesized, and the expression levels of mitochondrial respiration complexes were decreased in $206 \rho^{\circ}$ cells. We supposed that mitochondrial respiration dysfunction would be exhibited in $206 \rho^{\circ}$ cells. The Seahorse assay ${ }^{16}$ results demonstrated completely reduced respiratory chain function and increased non-mitochondrial respiration in $206 \rho^{\circ}$ cells (Figure 2). This result indicated that
$206 \rho^{\circ}$ cells produced ATP that primarily relied on nonmitochondrial respiration after mitochondrial dysfunction.

\section{Mitochondrial morphological alterations}

Mitochondrial biochemical abnormalities in $206 \rho^{\circ}$ cells were confirmed. We reasoned whether the presence morphological alterations in $206 \rho^{\circ}$ cells. Transmission electron microscopy (TEM) images showed that the numbers of mitochondria were increased in $206 \rho^{\circ}$ cells, the size of mitochondria was smaller and their mitochondria exhibited that the cristae was broken, internal membranes were disorganized, outer membrane was clumped and detached, and mitochondrial matrix was reduced with large empty vacuoles in $206 \rho^{\circ}$ cells (Supplementary Figure S4).

The presence of Ago2 within the mitochondria

It was reported that various miRNAs were detected in isolated mitochondria from rat liver, rat ventricular myocytes and several common human cell lines, ${ }^{9}$ we anticipated that the increased miRNAs, targeting to the mitochondrial RNAs, might account

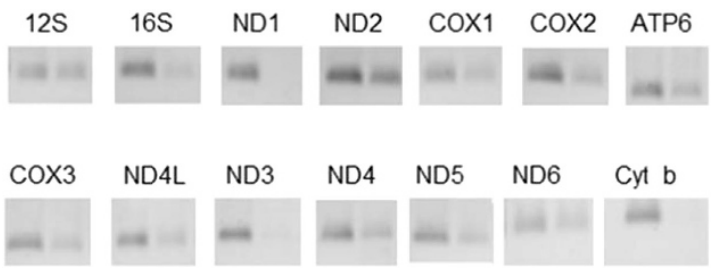

Figure 1. The assay of mitochondrial RNA expression efficiency by RT-PCR. Left bands are from 143B mt-R RNA CDNA and right bands are from 143B-206 $\rho^{\circ} \mathrm{mt}-\mathrm{R}$ cDNA.
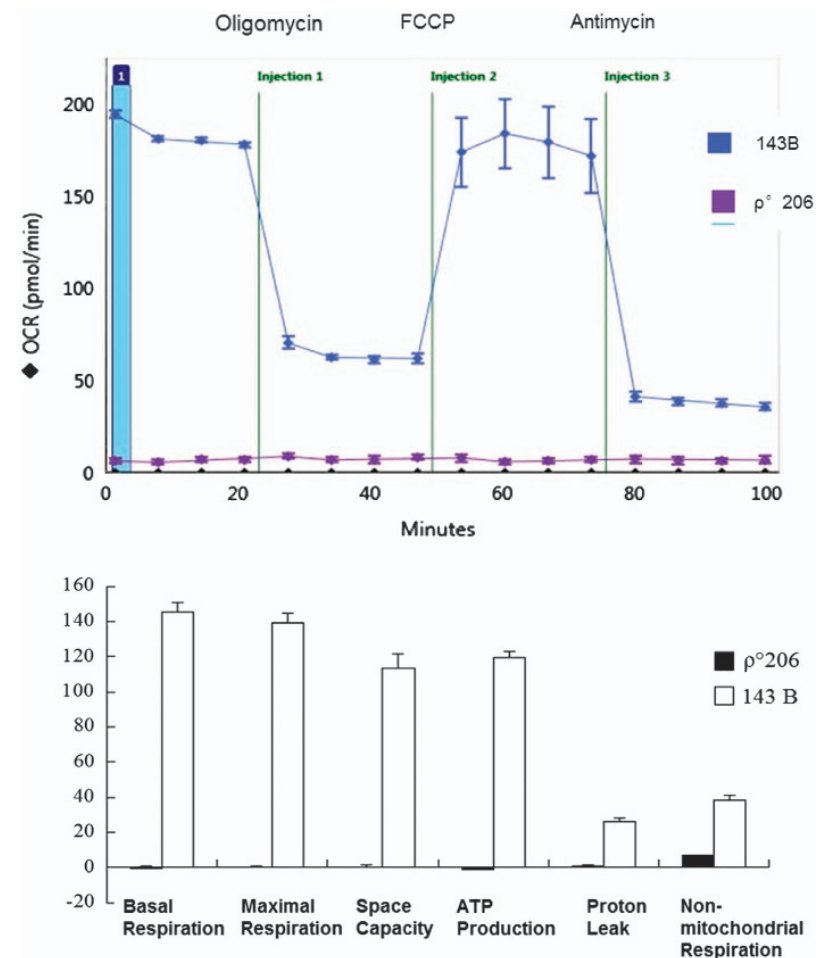

Figure 2. Mitochondrial function assay of $143 \mathrm{~B}$ and $143 \mathrm{~B}-206 \rho^{\circ}$ cells by Seahorse XF 24 analyzer. The bioenergetic profiles of $143 \mathrm{~B}$ and 143B-206 $\rho^{\circ}$ cells were generated by injecting electron transport chain inhibitors: oligomycin, FCCP and antimycin A to assess mitochondrial respiration. The results indicated mitochondrial dysfunction in 143B-206 $\rho^{\circ}$ cells. Non-mitochondrial respiration was kept in 143B-206 $\rho^{\circ}$ cells. 
for decreased translation in $206 \rho^{\circ}$ cell mitochondria. To begin exploring these possibilities, we first used highly purified mitochondria (Figure 3a) to demonstrate whether a fraction of Ago2 was present within the mitochondria. The results demonstrated that Ago 2 was indeed within $\mathrm{mt}$ and $\mathrm{mt}-\mathrm{R}$ from $143 \mathrm{~B}$ and $206 \rho^{\circ}$ cells (Figure $3 b$ ). Ago 2 was detected to be highly expressed in $206 \rho^{\circ} \mathrm{mt}$ and $\mathrm{mt}-\mathrm{R}$ samples (Figure $3 \mathrm{~b}$ ).

miRNA profile of mtDNA-less cells

The key component of RISC, Ago2, was within cell mitochondria of $143 \mathrm{~B}$ and $206 \rho^{\circ}$ cells. RISC should be within their mitochondria and regulate mitochondrial RNAs. Several miRNAs were detected in mitochondria. ${ }^{8,9,13}$ However, the functional significance of miRNAs in the mitochondria has remained largely unknown, especially in mtDNA-less cells.

Mitochondria-enriched miRNAs. To determine whether miRNAs were in mitochondria of $143 \mathrm{~B}$ and $206 \rho^{\circ}$ cells, we performed next-generation sequencing of small RNA (sRNA) using their mt-R RNA samples, their C-p RNA and mt RNA samples were as control. sRNA patterns of C-p RNA and mt-R RNA from 143B and $206 \rho^{\circ}$ cells were shown in Supplementary Figure S5. By comparing $m t-R$ miRNA patterns $\left(\mathrm{Li}-30=143 \mathrm{~B} \mathrm{mt}-\mathrm{R} ; 206 \rho^{\circ} \mathrm{mt}-\mathrm{R}\right)$ with $\mathrm{C}-\mathrm{p}$ miRNA patterns (Li-29 $=143 B$ C-p; Li-27 $\left.=206 \rho^{\circ} \mathrm{C}-\mathrm{p}\right)$, we found that miRNAs in mt-R RNA samples were much less than in C-p RNA samples. The results indicated that miRNAs on outer membrane of mitochondria were digested by RNase $A$, and the miRNAs from $\mathrm{mt}-\mathrm{R}$ should be in mitochondria. The miRNA percentages of $\mathrm{C}-\mathrm{p}$
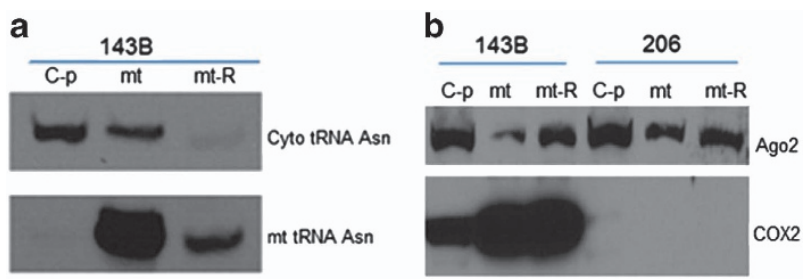

Figure 3. Pure mitochondria, Ago 2 within mitochondria. (a) Northern blotting was performed by using $\mathrm{mt}$ tRNA Asn probe and cytosolic tRNA Asn probe to check $C-p, m t$ and mt-R, which indicated that mitochondria were pure, and cyto tRNA was not present in mt-R. (b) Western blot results confirmed the presence of Ago2 within $143 \mathrm{~B}$ and $206 \rho^{\circ}$ mitochondria ( $\mathrm{mt}$ and $\mathrm{mt}-\mathrm{R}$ samples).
RNA samples from $143 \mathrm{~B}$ and $143 \mathrm{~B}-206 \rho^{\circ}$ cells were higher than the respective mt-R RNA samples (Supplementary Figure $S 6$ ). Owing to the extremely low amount of miRNAs in mt-R RNA from $206 \rho^{\circ}$ cells, we performed sRNA sequencing using $206 \rho^{\circ} \mathrm{mt}$ RNA sample in order to identify mitochondria-enriched miRNAs.

To identify mitochondria-enriched miRNAs, the miRNA ratios of mt-R RNA/C-p RNA from 143B and the miRNA ratios of mt-R RNA/ mt RNA from $206 \rho^{\circ}$ cells were calculated. The results demonstrated that some mature miRNAs were enriched in $143 \mathrm{~B}$ mitochondrial RNA and $206 \rho^{\circ}$ mitochondrial RNA. Thirty-nine miRNAs in $206 \rho^{\circ}$ mt-R RNA were enriched compared with $206 \rho^{\circ}$ mt RNAs including miR-133a, 499a-5p, 372, 1, 127-3p, 126-3p, $1305,501-3 p, 143-3 p, 887,122-5 p, 491-5 p, 146 a-5 p, 181 c-5 p$ and $145-5 p$ and so on (Supplementary Table S7). Twenty-eight miRNA in $143 \mathrm{~B}$ mt-R RNA were higher than C-p RNA including miR-4787$3 p, 4510,141-3 p, 203,200 c-3 p, 660-5 p, 181 d, 146 a-5 p, 181 c-5 p$, 99a-5p, 365a-3p, 365b-3p, 138-5p, 501-3p, 342-3p, 193b-3p, 99b-3p, let-7c, 181a-2-3p, 103a-3p, 19b-3p, 10b-5p, 125a-5p, 484, 148b-3p and 181a-5p (Supplementary Table S7).

From the miRNA ratios of $206 \rho^{\circ} \mathrm{mt}-\mathrm{R} / 143 \mathrm{~B} \mathrm{mt}-\mathrm{R}, 206 \rho^{\circ} \mathrm{C}-\mathrm{p} /$ 143B C-p and $206 \rho^{\circ} \mathrm{mt}-\mathrm{R} / 206 \rho^{\circ} \mathrm{mt}$, we found that miR-143-3p, $378 a-3 p, 146 a-5 p, 181 c-5 p$ and $501-3 p$ were presented in RNA samples of $206 \rho^{\circ} \mathrm{C}-\mathrm{p}, 206 \rho^{\circ} \mathrm{mt}, 206 \rho^{\circ} \mathrm{mt}-\mathrm{R}, 143 \mathrm{~B}$ C-p and 143B $m t-R$, respectively (Figure $4 a$ and Supplementary Table S7). These five miRNAs were more enriched in $206 \rho^{\circ}$ mt-R RNA than in $206 \rho^{\circ}$ mt RNA (Figure 4a and Supplementary Table S7). Highexpression levels of miR-181c-5p and 146a-5p in 206 mt-R RNA indicated that miR-181c-5p and $146 a-5 p$ might modulate mitochondrial RNAs in these cells.

miRNAs target on mitochondria-specific RNAs. Computational analyses of miRNA targets showed that miR-181c-5p had 23 and miR-146a-5p had 19 potential targets on mitochondrial mRNAs, rRNAs, tRNAs and non-coding regions (Table 1). The results indicated that miR-181c-5p and miR-146a-5p may inhibit mitochondrial RNAs to modulate mitochondrial gene expression. ${ }^{8,9,17}$

To determine whether miR-181c-5p and miR-146a-5p were enriched in $206 \rho^{\circ}$ cell mitochondria, we also performed RT-qPCR using miRNA cDNAs from 143B C-p RNA, $206 \rho^{\circ}$ C-p RNA and 206 $\rho^{\circ} \mathrm{mt}-\mathrm{R}$ RNA to validate our sRNA-sequencing data. The results demonstrated that miR-181c-5p and miR-146a-5p were highly enriched in $206 \rho^{\circ}$ mitochondrial fraction compared with control miR-423-5p (Figure 4b). a

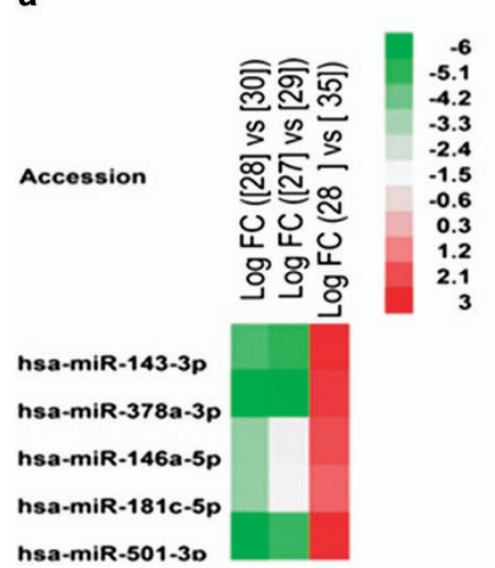

b

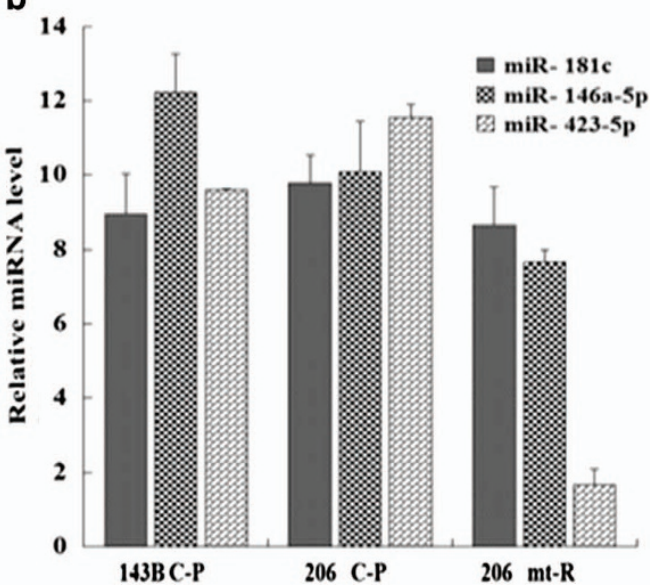

Figure 4. (a) Hotmap of mitochondria-enriched miRNAs. $30=143 \mathrm{~B}$ mt-R RNA, $28=206 \rho^{\circ} \mathrm{mt}-\mathrm{R}$ RNA, $29=143 \mathrm{~B} \mathrm{C}-\mathrm{p}$ RNA, $27=206 \rho^{\circ} \mathrm{C}-\mathrm{p}$ RNA and $35=206 \rho^{\circ} \mathrm{mt}$ RNA. (b) RT-qPCR results confirmed miR-181c-5p (miR-181c), miR-146a-5p were highly enriched in $206 \rho^{\circ}$ mitochondria $(\mathrm{mt}-\mathrm{R})$, the control miR-423-5p was not enriched in $206 \rho^{\circ}$ mitochondria. $5 \mathrm{~S}$ was as an internal control for quantification. 
would ameliorate the inhibition of miRNAs on mitochondria-

Table 1. miRNAs' potential targets on mitochondrial rRNAs, mRNAs, tRNAs and non-coding regions

\begin{tabular}{|c|c|c|}
\hline mt Gene & miR-181c-5p & miR-146a-5p \\
\hline 12S RNA & $\begin{array}{c}978-984 \\
1229-1235\end{array}$ & \\
\hline 16S RNA & $2320-2326$ & $\begin{array}{l}2245-2251 \\
2361-2367\end{array}$ \\
\hline ND1 & $\begin{array}{l}3593-3599 \\
3599-3605 \\
4191-4197\end{array}$ & $\begin{array}{l}3309-3316 \\
4120-4126\end{array}$ \\
\hline ND2 & & $\begin{array}{l}4594-4600 \\
5044-5050 \\
5198-5205 \\
5274-5280\end{array}$ \\
\hline ND4 & $\begin{array}{l}11083-11090 \\
11282-11288 \\
12013-12019\end{array}$ & $11889-11895$ \\
\hline ND5 & $\begin{array}{l}12840-12846 \\
13443-13450 \\
13495-13501 \\
13635-13641\end{array}$ & $\begin{array}{l}12528-12534 \\
12681-12688\end{array}$ \\
\hline ND6 & $\begin{array}{l}14199-14205 \\
14401-14407\end{array}$ & $\begin{array}{l}14517-14523 \\
14538-14544\end{array}$ \\
\hline $\operatorname{cox} 1$ & $6273-6282$ & \\
\hline $\operatorname{cox} 2$ & 7915-7921 & \\
\hline $\mathrm{COX} 3$ & 9425-9431 & \\
\hline ATP6 & 8976-8984 & \\
\hline ATP8 & & $8516-8522$ \\
\hline Cytb & $14910-14916$ & \\
\hline tRNA Ala & & $\begin{array}{l}5615-5621 \\
4347-4353\end{array}$ \\
\hline $\begin{array}{l}\text { tRNA Glu } \\
\text { tRNA Gly }\end{array}$ & $10041-10049$ & 4347-4353 \\
\hline $\begin{array}{l}\text { tRNA Ser(UCN) } \\
\text { tRNA Ser(AGY) }\end{array}$ & & $\begin{array}{c}7492-7498 \\
12220-12226\end{array}$ \\
\hline D-loop & $\begin{array}{c}245-252 \\
16066-16072\end{array}$ & $16364-16371$ \\
\hline
\end{tabular}

miRNAs target on mitochondria-associated mRNAs encoded by nucleus. More than 1500 genes are associated with mitochondrial structure and/or function. ${ }^{11}$ The majorities of these genes are encoded by nucleus. miRNAs may target on mitochondriaassociated RNAs encoded by nuclear genes to regulate mitochondrial metabolism and mitochondrial function. There are 892 predicted targets for hsa-miR-181c-5p and 224 predicted targets for hsa-miR-146a-5p in miRNA database. Some targets are mitochondria-associated mRNAs, such as miR-181c-5p targets on SLC25A37, SLC7A11, SLC4A8, SLC4A10, MICU3, GPD1L, KCNQ5 and CAMK2D. miR-146a-5p targets on SLC10A3, SLC38A1, SCN3B, KCNJ16 (potassium inwardly rectifying channel, subfamily J, member 16), KCNA6 and CASK and so on.

miR-181c-5p and miR-146a-5p were mitochondria-enriched miRNAs in $206 \rho^{\circ}$ mitochondria. However, the expression levels of some miRNAs were low in $206 \rho^{\circ}$ mitochondria. From miRNA ratios of $206 \rho^{\circ} \mathrm{mt}-\mathrm{R} / 206 \rho^{\circ} \mathrm{mt}$ RNA, we found that low-expressed miRNAs in $206 \rho^{\circ}$ cell mitochondria included miR-155-5p, 196-5p, $128,148 b-3 p$, let-7b-5p, let-7a-5p, 197-3p,18a-5p, 15b-5p and so on (Supplementary Table S8). These miRNAs had targets on complex $V$ component mRNAs such as miR-155-5p targeted on ATP2C1, miR-128 on ATP8A1, miR-148b-3p on ATP11A and ATP8A1, let-7b-5p and let-7a-5p on ATP2B2, ATP2B4 and ATP2C2, miR-197-3p on ATP6V1A, miR-18a-5p on ATP2C2 and ATP8A1, and miR-15b-5p on ATP13A3 and ATP1B4 mRNA. In the meantime, several miRNAs targeted on mRNAs related to mitochondrial fission, fusion or RISC proteins, including miR-196a-5p targeted on OPA 1 and OPA3, miR-18a-5p on HSF 2 and miR-15b-5p on HSPA4L mRNA. These low-expressed miRNAs in $206 \rho^{\circ}$ mitochondria

associated mRNAs, and benefit for ATP production and mitochondrial dynamic change.

\section{DISCUSSION}

Mitochondrial dysfunction and compensation

EtBr has been shown to have effects on mammalian mtDNA replication. Relatively low concentrations of $\operatorname{EtBr}(0.1-2 \mu \mathrm{g} / \mathrm{ml})$ result in either partial or complete inhibition of mtDNA replication, but have no effect on the replication of nuclear DNA. ${ }^{12}$ The mtDNA-less $206 \rho^{\circ}$ cells were derived from $143 B$ cell line using low concentration of EtBr. ${ }^{12}$ We found low mtDNA copy number and partial expression of mitochondrial RNAs in mtDNA-less $206 \rho^{\circ}$ cells. But no 13 mitochondria-specific peptides were synthesized in $206 \rho^{\circ}$ cells. Compared with 143B, mitochondrial complexes V, III and II were 86.5, 29.4 and $49.4 \%$ respectively, and complexes I and IV were completely lost in $206 \rho^{\circ}$ cells. No mitochondrial ND1 and ND3 mRNAs expressed in $206 \rho^{\circ}$ cells may account for their loss of complex I. No mitochondrial cyto b mRNA may cause their complex III to decrease. Low expression of COX1, COX2 and COX3 may result in complex IV loss.

It is possible to maintain human cells that lack mtDNA, and thus lack mitochondrial respiration. Because of the absence of respiratory chain, $206 \rho^{\circ}$ cells develop growth requirements for pyrimidines and pyruvate. Uridine is a pyrimidine nucleotide that is required for synthesis of RNA and DNA. ${ }^{18}$ If there is no uridine in the culture medium, mtDNA-less $206 \rho^{\circ}$ cells will die in 4 days. Our results from Seahorse indicate that the respiration of mtDNA-less $206 \rho^{\circ}$ cells is non-mitochondrial respiration. These cells produce ATP from pyruvate, from glycolysis of glucose and from medium supplement to generate acetyl-CoA into Krebs cycle in the matrix of mitochondria. Therefore, uridine and pyruvate in culture medium are necessary supplements for mtDNA-less cell growth.

Another reason to maintain the growth of mtDNA-less $206 \rho^{\circ}$ cells is compensation mechanisms. The first is the increased mitochondria numbers in $206 \rho^{\circ}$ cells (Supplementary Figure S4) to increase respiration volume. Also, high-activity complex $\mathrm{V}$ ( $86.5 \%$ of $143 B$ cell) benefits to generate ATP efficiently.

miRNAs target on mitochondrial mRNAs

Mitochondrial RNAs of ND1, ND3 and cyto b did not express in 206 $\rho^{\circ}$ cells, whereas the expression of other RNAs including 12S, 16S, ND2. COX1, COX2, ATP6, COX3, ND4L, ND4, ND5 and ND6 was low in $206 \rho^{\circ}$ cells compared with $143 \mathrm{~B}$ cells. The decreased mitochondrial RNA expression is the result from low copy of mtDNA in $206 \rho^{\circ}$ cells (mtDNA is $1 / 164$ of $143 \mathrm{~B}$ cells). But unequal transcription of mitochondrial RNAs (Figure 1) may indicate the post-transcriptional cleavage and processing mechanisms in the regulation of mitochondrial gene expression. ${ }^{19}$ miRNAs, as important post-transcriptional regulators of gene expression in human cells, may modulate these mitochondrial RNAs expression. ${ }^{8,9}$ It is confirmed that several miRNAs are translocated into mitochondria and modulate specific mitochondrial genomeencoded transcripts. ${ }^{89}$ The mature miRNA is incorporated into RISC, which recognizes target mRNAs through imperfect base pairing with the miRNA, and most commonly results in translational inhibition or destabilization of the target mRNA. Ago2 is highly expressed in $206 \rho^{\circ}$ cell mitochondria, which indicate that RISC is within $206 \rho^{\circ}$ cell mitochondria, and miRNAs regulate mitochondrial RNAs.

sRNA sequencing and $\mathrm{QPCR}$ analysis data confirmed that miR-181c-5p and miR-146a-5p were enriched in $206 \rho^{\circ}$ cell mitochondria. These two miRNAs have multiple targets on mitochondrial RNAs (Table 1). miR-181c-5p has 23 potential targets on mitochondrial RNAs including $12 \mathrm{~S}$ rRNA, 16S rRNA, ND1, ND4, ND5, ND6, COX1-3, ATP6 and Ctyb mRNA. miR-181c has 
been confirmed to be imported into rat mitochondria and repressed rat mitochondrial COX1 expression. miR-181c has multiple potential targets (Table 1), except COX1 mRNA, and may target other mitochondrial mRNAs, rRNAs and tRNAs.

miR-146a-5p has 19 potential targets on mitochondrial RNAs including 16S rRNA, ND1, ND2, ND4, ND5, ND6 and Cytb mRNAs (Table 1). RT-PCR showed that several mitochondrial RNAs were partially expressed in $206 \rho^{\circ}$ cells, but 13 of mitochondria-specific protein in $206 \rho^{\circ}$ cells are lack. Lack of 13 of mitochondrial mRNA translation may result from mitochondrial ribosomal RNA (16S rRNA and/or $12 \mathrm{~S}$ rRNA) dysfunction or due to miRNAs (for example miR-181c-5p and miR-146a-5p) cut mitochondrial mRNAs or suppress mitochondrial mRNA translation.

miRNAs may target on mitochondria-associated nuclear mRNAs. Both miR-181c-5p and miR-146a-5p target on SLC mRNAs. SLC25A has important role in mitochondrial fission. These two miRNAs target on ion channel and calcium transfer mRNAs encoded by nucleus, such as KCNQ5, KCNJ16, KCNA6, SCN3B, CAMK2D and CASK. The increase of miR-181c-5p and miR-146a-5p may downregulate these mitochondria-associated nuclear mRNAs to maintain the stabilization of mitochondrial membrane potential and calcium balance.

However, the expression of several miRNAs including miR-1555p, 196-5p, 128, 148b-3p, let-7b-5p, let-7a-5p, 197-3p,18a-5p and $15 b-5 p$ was low in $206 \rho^{\circ}$ mitochondria. These miRNAs were inhibitors of ATPase and mitochondrial fission and fusion mRNAs. Low expression of these miRNAs would improve the efficiency of ATP generation and mitochondria dynamic change. Nonmitochondrial respiration and the increase in the numbers of mitochondria in $206 \rho^{\circ}$ may be the result of these low-expressed miRNAs.

This is the first study to investigate the molecular, metabolic and morphological abnormalities, as well as miRNA profile in mtDNA-less $206 \rho^{\circ}$ cells. Several mitochondria-enriched miRNAs (including miR-181c-5p and miR-146a-5p) were identified in $206 \rho^{\circ}$ cell mitochondria. These two miRNAs targeted on multiple mitochondria-specific RNAs including mitochondrial mRNA, rRNA and tRNAs, as well as on nucleus-encoded mitochondriaassociated mRNAs. This study provided direct evidence that miRNAs were imported into mitochondria and regulated mitochondrial RNAs in mtDNA-less cells.

\section{MATERIALS AND METHODS}

\section{Cell lines}

$143 B$ cells were grown in DMEM (Invitrogen) containing glucose $(4.5 \mathrm{~g} / \mathrm{l})$, completed with $10 \%$ fetal bovine serum, $0.1 \mathrm{mg} / \mathrm{ml}$ sodium pyruvate and $100 \mathrm{U} / \mathrm{ml}$ penicillin-streptomycin. mtDNA-less $206 \rho^{\circ}$ cells were cultured with same medium supplement with $50 \mu \mathrm{g} / \mathrm{ml}$ urine. Cell pellets $(C-p)$ were collected from one $10-\mathrm{cm}$ dish of $143 \mathrm{~B}$ and $206 \rho^{\circ}$ cells, then washed two times by PBS.

\section{Analysis of mitochondrial genome}

Genomic DNA was isolated from 143B C-p and $206 \rho^{\circ}$ C-p samples. The entire mitochondrial genome of $143 B$ and $206 \rho^{\circ}$ cells was PCR-amplified in 24 overlapping fragments by using sets of the light- and the heavystrand oligonucleotide primers, as described previously. ${ }^{14,20}$ These PCR amplicons were purified and subsequently analyzed by direct sequencing. The BLAST homology searches were performed using the programs available on the NCBI website. ${ }^{15}$ The relative DNA copy numbers of $206 \rho^{\circ}$ cells to $143 \mathrm{~B}$ cells was performed by qPCR. The ratio of mtDNA HV1 region to nucleus-encoded $H 3$ gene was determined as the relative DNA copy numbers of $206 \rho^{\circ}$ cells. PCR primers for amplification of $H 3$ and $H V 1$ genes were listed in Supplementary Table S9. ${ }^{9}$

Examination of mitochondria purity and Ago2

$143 B$ and $206 \rho^{\circ}$ cellular mitochondria were isolated with the Mitochondrial Isolation kit (Miltenyi Biotec, MACS, Auburn, CA, USA) and monoclonal
anti-TOM22-conjugated microbeads, as described, with several modifications. ${ }^{21}$ Theses mitochondrial pellets (called $\mathrm{mt}$ ) were treated with RNase A treated (mt-R) for mitochondrial RNA isolation. After determination of mitochondrial purity, we used these samples to examine whether Ago 2 was present within mitochondria from 143B and $206 \rho^{\circ}$ cells by western blotting using monoclonal Anti-Ago2 antibody (SAB4200085, Sigma-Aldrich, St. Louis, MO, USA).

\section{Analysis of mitochondrial RNA expression}

RNA preparations from $143 \mathrm{~B}$ and $206 \rho^{\circ} \mathrm{C}-\mathrm{p}$, mt and $\mathrm{mt}-\mathrm{R}$ were obtained by using the Totally RNA kit (Ambion, Austin, TX, USA). ${ }^{22,23}$ RNAs (mRNA and miRNA) were extracted using the Ambion mirVana miRNA Isolation Kit (Ambion, AM1560) following manufacturer's instruction. First-strand cDNA was synthesized from $1 \mu \mathrm{g}$ of total RNA using miScript II RT kit (Qiagen, Valencia, CA, USA; 218161) for miRNA CDNA and mRNA cDNA, according to manufacturer's instructions. RT-qPCR (QuantiFast SYBER Green PCR kit, Qiagen, 204054) was carried out in Applied BioSystems 7500 Real Time PCR machine Applied Biosystems (Foster City, CA, USA) for quantification of mRNAs and miRNAs using these RNA samples from 143B and $206 \rho^{\circ}$ cell lines. 143B mitochondrial RNA expression was used as control. The mitochondria-specific primers for RT-PCR and RT-qPCR were listed in Supplementary Table S10.

\section{Assay of mitochondrial proteins}

The reliable method to determine whether $206 \rho^{\circ}$ cells translate mitochondrial peptides is to examine mitochondrial specific 13 peptides using $\left[{ }^{35} \mathrm{~S}\right]$-methionine- $\left[{ }^{35} \mathrm{~S}\right]$ cysteine pulse labeling of mitochondrial protein synthesis in vitro after blocking cytoplasmic protein synthesis by emetine as we described. ${ }^{22,23}$ Cellular protein samples from mtDNA-less $206 \rho^{\circ}$ cell line and controls of 143B cells and lymphoblastoid cell line were used.

To assay the mitochondrial complexes I-V in $206 \rho^{\circ}$ cells, SDS-PAGE and western blotting of $143 \mathrm{~B}$ and $\mathrm{mtDNA}$-less $206 \rho^{\circ}$ cell homogenates $(20 \mu \mathrm{g}$ of protein), samples were probed for mitochondrial respiratory chain complexes via total human OXPHOS WB antibody cocktail (Abcam, Cambridge, MA, USA; ab11041).

\section{Examination of mitochondrial morphology}

C-p samples from $143 \mathrm{~B}$ and $206 \rho^{\circ}$ cells were fixed for $24 \mathrm{~h}$ using fixative ( $2 \%$ glutaraldehyde ( $\mathrm{pH} 7.2$ ) and $0.1 \mathrm{M}$ cacodylate buffer $(\mathrm{pH} 7.4)$ ), then the samples were sent to Pathology Department in CCHMC to treat with 1-2\% osmium tetraoxide (OsO4), dehydrated, embedded in resin, sliced into ultra-thin sections, double-stained with uranyl acetate and lead citrate, and finally performed TEM examination.

\section{Measurements of mitochondrial respiration}

The cultured $143 \mathrm{~B}$ and $206 \rho^{\circ}$ cells were transferred to XF96 assay plate at 10,000 cells per well and were allowed grow overnight. ATP production and oxygen consumption were determined by XF96 Extracellular Flux Analyzers (Seahorse Biosciences, North Billerica, MA, USA). The parameters included basal respiration, ATP production, maximal respiratory capacity and proton leak. The respiration parameters were normalized by cell numbers. ${ }^{16}$

\section{SRNA sequencing}

RNA samples from 143B and $206 \rho^{\circ} \mathrm{C}-\mathrm{p}$, mt and mt-R were obtained by using the Totally RNA kit (Ambion). ${ }^{22}$ RNA purity was detected by our designed probes using C-p RNA, mt RNA and mt-R RNA samples. After the determination of purity of mt-R RNA from mitochondria, these RNA samples were studied by next-generation sequencing of sRNA to examine mitochondria-enriched miRNAs in $143 \mathrm{~B}$ and $206 \rho^{\circ}$ mitochondria. Nextgeneration sequencing of sRNA (15-49 bp), including miRNAs (18-23 bp), was carried out in The Genetic Variation and Gene Discovery Core in CCHMC (Cincinnati, OH, USA) using 500 ng C-p RNA of 143B and 206, as well as their $\mathrm{mt}$ and $\mathrm{mt}-\mathrm{R}$ RNA samples.

\section{Computational analyses of miRNA targets}

To scan miRNA's potential target sites on the mitochondrial RNAs, we performed a BLAST search ${ }^{15}$ of mitochondrial genome sequence and mature sequences of miR-181c-5p or miR-146a-5p. In addition, we used 
the target Scan algorithm (http://www.targetscan.org) to search for the presence of conserved target sites that match the seed region of miR$181 c-5 p$ and miR146a-5p, and assessed the structural accessibility of the predicted target site. ${ }^{24}$ The targets of miR-181c-5p, 146a-5p on nucleusencoded mitochondrial genes were obtained by searching miRNA database (http://www.mirbase.org).

Verification of mitochondria-enriched miRNAs

To determine whether miR-181c-5p and miR-146a-5p enriched in $206 \rho^{\circ}$ cell mitochondria, the miRNA cDNA synthesized from 143B C-p RNA, $206 \rho^{\circ}$ C-p RNA and $206 \rho^{\circ} \mathrm{mt}-\mathrm{R}$ RNA samples were used to do RT-qPCR to quantify miR-181c-5p and miR-146a-5p in C-p and in mitochondria. miR-423-5p was used as control.

\section{ACKNOWLEDGEMENTS}

We are grateful to Anne Chomyn (California Institute of Technology) and Min-xin Guan (Cincinnati Children's Hospital Medical Center) for 143B and $206 \rho^{\circ}$ cell lines. This work was supported by Divisional Start-up package in Human Genetics Division in Cincinnati Children's Hospital Medical Center for RL.

\section{COMPETING INTERESTS}

The authors declare no conflict of interest.

\section{REFERENCES}

1 Laffont B, Corduan A, Plé H, Duchez AC, Cloutier N, Boilard E et al. Activated platelets can deliver mRNA regulatory Ago2-microRNA complexes to endothelia cells via microparticles. Blood 2013; 122: 253-261.

2 Beitzinger M, Peters L, Zhu JY, Kremmer E, Meister G. Identification of human microRNA targets from isolated Argonaute protein complexes. RNA Biol 2007; 4 76-84.

3 Calin GA, Croce CM. MicroRNA signatures in human cancers. Nat Rev Cancer 2007; 6: 857-866.

4 Wang D, Zhang H, Li M, Frid MG, Flockton AR, McKeon BA et al. MicroRNA-124 controls the proliferative, migratory, and inflammatory phenotype of pulmonary vascular fibroblasts. Circ Res 2014; 114: 67-78.

5 Chen CZ, Li L, Lodish HF, Bartel DP. MicroRNAs modulate hematopoietic lineage differentiation. Science 2004; 303: 83-86.

6 Henao-Mejia J, Williams A, Goff LA, Staron M, Licona-Limón P, Kaech SM et al. microRNA miR-181 is a critical cellular metabolic rheostat essential for NKT cell ontogenesis and lymphocyte development and homeostasis. Immunity 2013; 38 : 984-997.

7 Rawal S, Manning P, Katare R. Cardiovascular microRNAs: as modulators and diagnostic biomarkers of diabetic heart disease. Cardiovasc Diabetol 2014 13: 44 .

8 Das S, Ferlito M, Kent OA, Fox-Talbot K, Wang R, Liu D et al. Nuclear miRNA regulates the mitochondrial genome in the heart. Circ Res 2012; 110: 1596-1603.
9 Zhang X, Zuo X, Yang B, Li Z, Xue Y, Zhou Y et al. MicroRNA directly enhances mitochondrial translation during muscle differentiation. Cell 2014; 158: 607-619.

10 Wallance DC. Mitochondrial diseases in man and mouse. Science 1999; 283: 1482-1488.

11 Calvo S, Jain M, Xie X, Sheth SA, Chang B, Goldberger OA et al. Systematic identification of human mitochondrial disease genes through integrative genomics. Nat Genet 2006; 38: 576-582.

12 King MP, Attardi G. Human cells lacking mtDNA: repopulation with exogenous mitochondria by complementation. Science 1989; 246: 500-503.

13 Sripada L, Tomar D, Singh R. Mitochondria: one of the destinations of miRNAs Mitochondrion 2012; 12: 593-599.

14 Rieder MJ, Taylor SL, Tobe VO, Nickerson DA. Automating the identification of DNA variations using quality-based fluorescence re-sequencing: analysis of the human mitochondrial genome. Nucleic Acids Res 1998; 26: 967-973.

15 Yang L, Tan Z, Wang D, Xue L, Guan M, Huang T et al. Species identification through mitochondrial rRNA genetic analysis. Sci Rep 2014; 4: 4089.

16 Rogers GW, Brand MD, Petrosyan S, Ashok D, Elorza AA, Ferrick DA et al. High throughput microplate respiratory measurements using minimal quantities of isolated mitochondria. PLoS One 2011; 6: e21746.

17 Tomasetti M, Neuzil J, Dong L. MicroRNAs as regulators of mitochondrial function: role in cancer suppression. Biochim Biophys Acta 2014; 1840: 1441-1453.

18 Bismuth G, Thuillier L, Perignon JL, Cartier PH. Uridine as the only alternative to pyrimidine de novo synthesis in rat T lymphocytes. FEBS Lett 1982; 148 135-139.

19 Mercer TR, Neph S, Dinger ME, Crawford J, Smith MA, Shearwood AM et al. The human mitochondrial transcriptome. Cell 2011; 146: 645-658.

20 Li R, Xing G, Yan M, Cao X, Liu XZ, Bu X, Guan MX. Cosegregation of C-insertion at position 961 with the A1555G mutation of the mitochondrial 12 S rRNA gene in a large Chinese family with maternally inherited hearing loss. Am J Med Genet 2004 124A: 113-117.

21 Xu Y-H, Xu K, Sun Y, Liou B, Quinn B, Li R et al. Multiple pathogenic proteins implicated in neuronopathic Gaucher disease mice. Hum Mol Genet 2014; 23: 3943-3957.

22 Li R, Guan MX. Human mitochondrial leucyl-tRNA synthetase corrects mitochondrial dysfunctions due to the tRNALeu(UUR) A3243G mutation, associated with mitochondrial encephalomyopathy, lactic acidosis, and stroke-like symptoms and diabetes. Mol Cell Biol 2010; 30: 2147-2154.

23 Liu Y, Li R, Li Z, Wang X, Yang L, Wang $S$ et al. The mitochondrial transfer RNAMet 4435A_G mutation is associated with maternally hypertension in a Chinese pedigree. Hypertension 2009; 53: 1083-1090.

24 Bentwich I. Prediction and validation of microRNAs and their targets. FEBS Lett 2005; 579: 5904-5910.

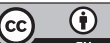

This work is licensed under a Creative Commons Attribution 4.0 International License. The images or other third party material in this article are included in the article's Creative Commons license, unless indicated otherwise in the credit line; if the material is not included under the Creative Commons license, users will need to obtain permission from the license holder to reproduce the material. To view a copy of this license, visit http://creativecommons.org/licenses/ by/4.0/

Supplemental Information accompanies the paper on the Cell Death Discovery website (http://www.nature.com/cddiscovery) 